\title{
PERSPECTIVA SOBRE POLÍTICAS PÚBLICAS DOS ATORES EM UM DESTINO TURÍSTICO: CASO DE CIUDAD JUÁREZ, CHIHUAHUA
}

\author{
Perspectiva sobre políticas públicas de los actores en un destino turístico: \\ Caso de Ciudad Juárez, Chihuahua \\ Perspective on public policies of the actors in a tourist destination: Case of
Ciudad Juarez, Chihuahua
}
Silvia Gabriela Verdugo González ${ }^{1}$ Isabel Zizaldra Hernández ${ }^{2}$ Zyanya Villa Zamorano ${ }^{3}$

Resumo: O manuscrito explora interações entre os atores do turismo e as políticas públicas (PP) com o patrimônio cultural e o desarrollo em Ciudad Juárez, Chihuahua (CJS). No México, como país adotou uma postura congruente no seu PP. Mientras, no CJS, existe ausencia em acessos pelo beneficio do destino. Realize uma aproximação do fenômeno usando a teoria do ator vermelho. Assim, a presente investigação tem um enfoque qualitativo. Veja a perspectiva dos atores sociais envolvidos no destino do turismo usando uma acomodação na realidade usando a experiência vivida. Se você localizar os atores e atores envolvidos na atividade em grupos focados em fevereiro de 2019. Atreves grupos focados, interpreta o fenômeno social, aplica uma guia com cinco instruções, os depoimentos são processados com o software Atlas.Ti. Os mais diversos possíveis benefícios para o CJS serão evidenciados na medida em que existem políticas públicas no turismo que afetam o patrimônio cultural.

Palavras Chave: Turismo; Políticas Públicas; Teoria do ator-vermelho; Patrimônio Cultural; Norte do México.

Resumen: El manuscrito explora interacciones de los actores del turismo y las políticas públicas (PP) con enfoque en el patrimonio cultural y el desarrollo en Ciudad Juárez, Chihuahua (CJS). En México como país muestra una postura congruente en sus PP. Mientras, en CJS existe ausencia en acciones por el benefício del destino. Se realiza una aproximación al fenómeno mediante la teoría red-actor. Así, la presente investigación tiene un enfoque cualitativo. Se examina la perspectiva de los actores sociales involucrados en el destino de turismo mediante un acercamiento a la realidad mediante la experiencia vivida. Se convocó a los actores e interesados en la actividad en grupos focales diferidos entre febrero-junio de 2019. Atreves de grupos focales, se interpreta el fenómeno social, aplicado

\footnotetext{
${ }^{1}$ Licenciada en Turismo, Universidad Autónoma de Ciudad Juárez. Universidad Autónoma de Ciudad Juárez (UACJ). E-mail: http://www.uacj.mx/ Orcid: https://orcid.org/0000-0002-6765-4346

${ }^{2}$ Doctorada en Turisme, Dret e Empresa, Universitat de Girona (UdG), España.

Maestra Investigadora, Profesora de Tiempo Completo (PTC) adscrita al Departamento de Ciencias Administrativas, Participa en el Doctorado en Ciencias Administrativas, Licenciatura en Turismo y Miembro del Cuerpo Académico de 31 Estudios sobre Turismo y Tiempo Libre en la Universidad Autónoma de Ciudad Juárez. Email: izizaldr@uaci.mx isabel.zizaldra@gmail.com Orcid: https://orcid.org/ 0000-0001-7079-5521

3 Maestra en Ciencias para el Desarrollo, Sustentabilidad y Turismo, Universidad Autónoma de Nayarit, México. E-mail: zyanya.villa.zamorano@gmail.com Orcid: https://orcid.org/0000-0002-90148556
} 


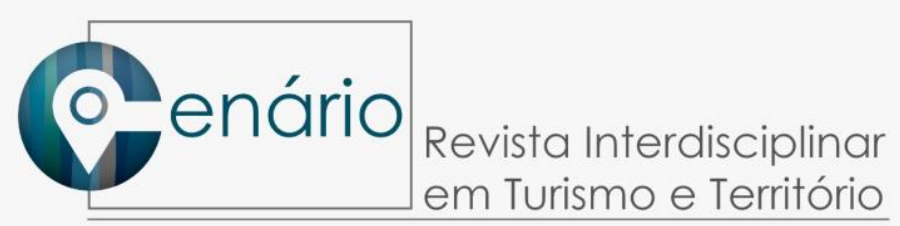

una guía con cinco preguntas, los testimonios se procesan con el software Atlas.Ti. Los hallazgos muestran posibles beneficios para CJS serán evidentes en la medida que existan políticas públicas en turismo que atiendan al patrimonio cultural.

Palabras Clave: Turismo; Políticas Públicas; Teoría del actor-red; Patrimonio Cultural; Norte de México.

Abstract: The manuscript explores interactions of tourism and public policy (PP) actors with a focus on cultural heritage and development in Ciudad Juárez, Chihuahua (CJS). In Mexico, like a country, it shows a consistent stance in its PP. Meanwhile, in CJS, there is an absence in shares for the benefit of fate. An approximation to the phenomenon is using the network actor theory. Thus, the present investigation has a qualitative approach. The perspective of the social actors involved in the tourism destination is examined through a plan to reality through lived experience. Actors and those interested in the activity in deferred focus groups were between February-June 2019. Through focus groups, the social phenomenon is interpreted, applying a guide with five questions, the testimonials processed with Atlas.Ti software. The findings show possible benefits for CJS will be evident to the extent that there are public tourism policies that address cultural heritage.

Keywords: Tourism; Public politics; Actor-network theory; Cultural heritage; North of Mexico.

\section{INTRODUCCIÓN}

El turismo es una actividad económica relevante para México por su aportación al producto interno bruto y a la balanza de pagos. Esto se ve reflejado en la obtención del 6to lugar por concepto de captación de turistas que se traducen en 21 mil millones de dólares por concepto de divisas y la generación de más de 4 millones de empleos relacionados a esta actividad terciaria (SECTUR y DATATUR, 2018).

Mientras la postura de México en política pública turística (PPT) es congruente en sus planes y programas. Son programas bien evaluados evalúalos y articulados, pues tienden a la estimulación de inversión, como al crecimiento. Ejemplos de lo anterior se encuentran primordialmente en los destinos de playa, como Ixtapa, Zihuatanejo, Los Cabos, o Cancún.

Aun con sus aportaciones a la economía del país, el turismo para el gobierno no puede ser visto solo como un impulsador de la economía, sino como afirma Magaña-Carrillo (2009), se debe considerar de importancia las implicaciones culturales y educativas que este brinda tanto a turistas como a la comunidad que los recibe.

No obstante, existe un divorcio entre la concepción del patrimonio cultural y el turismo. Al considerase que lo primero no debe ser trasgredido por la actividad. Es así como se requiere un plan de trabajo, con acciones que permitan visualización de acciones que generen beneficios cualitativos y cuantitativos, en este sentido medibles, es decir para el gobierno es la creación de política pública (OCDE, 2016). Este escenario es del ámbito público, ya que sus medidas permitirán un aprovechamiento de los recursos. Y en el caso del turismo afectan su actividad.

\section{PLANTEAMIENTO DEL PROBLEMA}

En Ciudad Juárez (CJS), a inicios del siglo XX, la actividad turística fue creciendo como alternativa para la comunidad, como consecuencia de la disminución de otros sectores económicos. Luego entonces, CJS ha sido antípoda por diferentes periodos relacionados con la visita al destino. Ejemplo de ello es la construcción de la Plaza de Toros entre 1903 - 


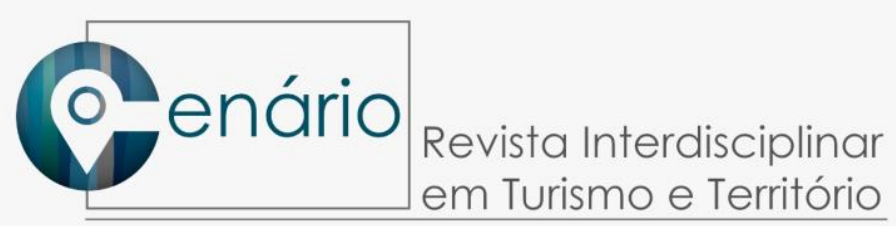

1905, ya que genero una oportunidad de diversificación económica (Zizaldra, 2010), asimismo para los años 20 con la prohibición del consumo de alcohol (Ley Seca) en Estados Unidos de Norteamérica (EUA), dio un impulso al visitante transfronterizo con la afluencia a bares, cantinas, garitos y centros nocturnos.

El enfoque ha sido diferenciado, pues la comunidad ha pasado por los Divorcios Exprés, la II Guerra Mundial, los legrados, las visitas por compras o por citas al médico. Como el Programa Nacional fronterizo (PRONAF), el cual se enfocaba en la atracción de turismo, para ello se amplió la infraestructura (museos; centros de artesanías; hoteles, teatros entre otros), obras públicas, que además prendía el apoyo a la zona libre. Pues las fronteras se veían como la puerta de entrada a México en norte del país.

De manera que, en CJS se impulsa el turismo de negocios y el de médico desde finales del siglo XX y comienzos del XXI. Ambas actividades han sido significativas para el destino. La primera influenciada por el ámbito de la manufactura instala a finales de los años 60's, y la cual cuenta con alrededor de 330 empresas y más de 291 mil empleados (Index, 2018). En cuanto al Turismo Médico, es se amplía al de salud y bienestar. Este representa un constante flujo, pues los procedimientos son más económicos y con calidad similar al de EUA. Apoyado, por una asistencia con calidad humana por profesionales en la salud.

Sin embargo, una desventaja para el destino ha sido la visión limitada de los tres niveles de gobierno para el apoyo en la actividad. Pues, CJS no cuentan con políticas públicas en el cuidado y conservación del patrimonio cultural y de la actividad. Pues: "Es difícil imaginar el desarrollo turístico de un destino sin políticas que se orienten es este sentido. Así la sustentabilidad y la competitividad van de la mano en un proceso que se retroalimenta permanentemente (Villar, 2009: 72)"

Es una falta de políticas con enfoque en el patrimonio cultural. Ante su ausencia, se refleja en las directrices, y con ello se pierde el sentido de identidad y amor hacia la ciudad. En este sentido la actividad inutiliza la orientación de una vocación del destino: "[...] los lugares turísticos son una forma de construir los discursos y figuras de la identidad nacional de una nación. En el caso de México [se utilizaron] el desarrollo de zonas turísticas como escenarios para ilustrar distintas partes del relato sobre la identidad mexicana que querían sostener". (Velázquez, 2013:107). Su ausencia se refleja en la sostenibilidad del destino, al no permear de manera correcta a los habitantes, y en consecuencia a los turistas.

De manera que, las PPT son la forma tangible de la acción del Estado y son la forma en la que se conecta la ciudadanía con el gobierno, pues se busca con ellas el bienestar colectivo, a través de designación de responsabilidades a los actores sociales (Torres-Melo y Santander, 2013; Villar, 2009). Ya que: "una política pública se compone de un conjunto de decisiones y acciones dirigidas a resolver un problema público. La solución de dicho problema dependerá no sólo de la idoneidad del diseño de la política pública, sino de su eficaz implementación (Cejudo y Michel (2016:4)".

Por tanto, los alcances de las PPT influyen en la ciudad, desde la perspectiva de los actores sociales involucrados. En este sentido, se plantean las siguientes interrogantes: ¿Si existen políticas públicas enfocadas al turismo en el destino?; ¿Qué actores son partícipes del desarrollo de políticas públicas?; ¿Cuáles son los beneficios de las políticas públicas en turismo que apoyen el patrimonio cultural?

El objetivo general se enmarca en: Explorar políticas públicas enfocadas al turismo de Ciudad Juárez que permiten la preservación del patrimonio cultural y el desarrollo económico. Mientras los objetivos específicos se centran en: Verificar el interés por políticas públicas en turismo y patrimonio cultural en los prestadores de servicios, gobierno e instituciones de educación; Desglosar beneficios que se tendrían por la protección del patrimonio cultural en Ciudad Juárez; e Indagar que políticas públicas existentes apoyan el patrimonio cultura y el turismo en Ciudad Juárez, a través de los actores sociales involucrados.

En consecuencia, los supuestos plantean: 


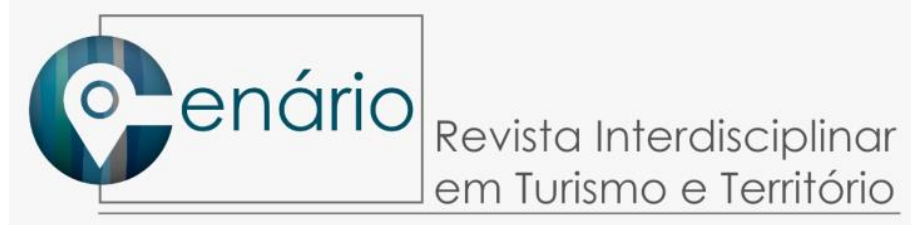

- $\quad$ S1. En Ciudad Juárez las políticas públicas en turismo con enfoque en el patrimonio cultural son escasas o inexistentes;

- $\quad$ S2. Existe bajo interés en el impulso de políticas públicas en turismo por actores e interesados en turismo.

\section{MARCO TEÓRICO}

El turismo es una actividad de crecimiento sostenido en términos globales. Su influencia en las naciones que lo han incorporado observan un beneficio en lo económico, más no necesariamente en el ámbito ambiental, sin embargo, repercute significativamente en la calidad de vida. Por lo anterior se ha elegido la teoría del actor-red (ANT por sus siglas en inglés y los conceptos de desarrollo, políticas públicas y turismo.

\section{Teoría del Actor- Red}

Actor-Network Theory o Teoría del Actor-Red es utilizada de forma incipiente como metodología en el estudio de los fenómenos turísticos. En esta teoría, uno de los representantes es Latour, quien considera que la red se transfiere por la interacción de los actores en la red. Así la red se comprende como el nodo con conexiones, asociaciones y articulaciones y al mismo tiempo están conectadas con otros nodos en perspectiva (no individual o unidireccional) (Bezerra, da Silva, de Assis, Dias, de Melo y Sávio, 2017).

El acercamiento a la teoría ANT, tiene una mayor significancia en la manera en que es observado un fenómeno. Para ello se debe superar lo interpretativo, con la finalidad de la búsqueda de lo descriptivo; asociar lo objetivo y lo subjetivo; cuestionar paradigmas, al igual de mostrar cómo los acontecimientos trastocan lo social y natural por igual. Se enfoca prioritariamente a lo que debe ser observado durante la investigación, es decir, más hacia el cómo, que hacia el qué. (Morales, 2015)

Las redes auto organizadas no necesariamente se dan mediante la planificación, estas surgen de forma espontánea (público, privado y tercer sector) y cada vez más ganan terreno. Y estas encuentra una cercanía con la trasformación social que está ocurriendo desde lo glocal (local a lo global). Teoría del Actor-Red aún tiene una restricción en cuanto a la capacidad del número de actores que el investigador puede articular y comprender desde una perspectiva social (Meyer and Bulgacov, 2014). En este orden de ideas, es un principio de proyección abstracta, es decir, no es una decisión concreta o arbitraria en las formas que deben estar representadas en el diagrama. Es decir:

\section{[...] se enfrenta a un mundo poblado de proposiciones. Un universo que se convierte en pluriverso en tanto que tales proposiciones son incorporadas a través del concepto. En ese sentido, nuestra tesis es que esta perspectiva no se comprende en toda su magnitud si no se atiende a este papel creativo (López y Tirado, 2012: 10)}

\section{Desarrollo turístico}

El desarrollo turístico se da cuando existen: "[...] ciertas pautas que se deben encaminar al mejoramiento de las estrategias que conllevan a un destino turístico planificado; [...] sobre cuáles deben ser dichas pautas y las relaciones que debe establecer la política turística con las otras políticas sectoriales (Enríquez, 2012: 425)". De manera que la importancia de las PPT han de ser diseñadas cuidadosamente, para establecer la relación entre la operación y la infraestructura existente que apoya a la actividad. Pues existe una conexión con los ámbitos sociales en su delineación (Ruiz, 2008; Enríquez, 2012; Montañés, 2012,) Ya que:

Por la especificidad del proceso productivo de la actividad turística, en el que la colaboración y las relaciones se convierten en un aspecto más que fundamental, prestar atención a las dinámicas reales de colaboración que 
se producen entre los diversos actores (ya sean públicos o privados) que operan en el territorio, es el elemento clave para comprender el papel que tiene la gestión activa en el desarrollo del turismo en un territorio. (Merinero y Zamora, 2009, pp. 220)

Como remarca la teoría ANT, se requiere un acercamiento a la realidad mediante la experiencia vivida, que permita hacer descripciones detalladas, de la mirada de los actores en su continuo movimiento como reflejo de la su vida entre espacio (Clifford, 1999, en Morales, 2015).

\section{Políticas Públicas, Patrimonio Cultural y Turismo}

Las políticas públicas son gestiones que previamente han de ser diagnosticadas por el ámbito gubernamental. La cuales tendrán objetivos de interés social con la finalidad de que exista un beneficio mutuo, es decir que influyan en el correcto desarrollo de un destino. Pues, se requiere considerar la política, así como la planificación, de manera que integre beneficios, económicos, políticos, culturales, e intelectuales, con orientación coherente en la sustentabilidad del turismo, ante el reto de los destinos y de los países, por la prosperidad y la paz y la mejorar en la calidad de vida local y global (Edgell, Swanson, 2013).

Por lo anterior, es mediante el Estado quien planifica, por tanto: "En el caso del turismo se trata de la planificación urbana y territorial, que organiza y regula el uso del suelo a la vez asigna determinadas características a los territorios bajo la jurisdicción (Villar, 2009: 53)" En este orden de ideas la OCDE (2016), recomienda en el caso de la política turística: a) Fortalecer la gobernanza del turismo mediante un enfoque de política más estratégico e integrado; b) Fortalecer la conectividad aérea desde mercados emisores con alto potencial para apoyar la diversificación de mercados; c) Desarrollar una sistema de transporte más integrado que facilite la movilidad del turista en el interior del país; d) Adaptar el modelo de desarrollo turístico para responder a las tendencias del mercado; e) Distribuir de mejor manera los beneficios del turismo y promover un crecimiento incluyente.

En este sentido, la determinación y establecimiento de políticas turísticas con enfoque cultural, palia los rezagos en el contexto formativo. De manera que se tienen diferentes formas de efectuarlo, como son: "[...] los foros de consulta y las líneas electrónicas [...] como una forma de participación ciudadana abierta y democrática, con el cual se diseña y crea el programa respectivo de turismo para gestar un desarrollo integral, argumentando que todas las inquietudes [...] (Castillo, Peñaloza, Tamayo, 2008).

Por tanto, se requiere meditar sobre las políticas públicas con enfoque en el turismo. Pues se enuncia como: "un conjunto de acciones que realiza el Estado en materia turística con el fin de fomentar el turismo interno y externo y las actividades ligadas a éste" (Méndez (1988: 35) desde sus inicios, hasta la actualidad. Además:

[...] las políticas establecidas para desarrollar la oferta, en sus renglones de infraestructura y estructura, fueron importantes para el desarrollo turístico nacional y su promoción en el exterior [...] pero se dio menos importancia a filosofías como la del servicio de calidad total, donde se contempla al capital humano como parte central (Magaña-Carillo, 2009: 538).

Aunque: "[...] una política pública orientada a la calidad debe ajustar las características del servicio, a las expectativas del turista. (Villar, 2009:69)”. De acuerdo a la orientación actual al visitante, este sería el escenario ideal.

\section{METODOLOGÍA}




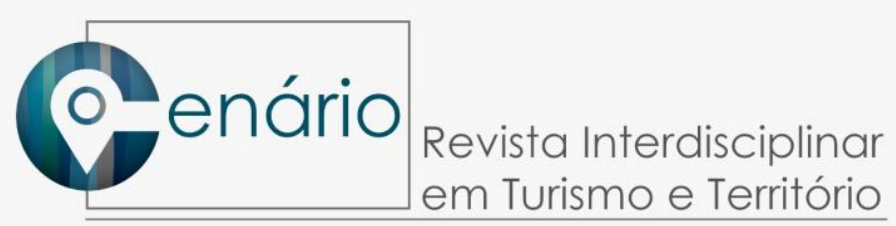

La presente investigación tiene un enfoque cualitativo, acorde con la interpretación de un fenómeno social, profundizando en el punto de vista y significados (Hernández, et al., 2013). Se recurre a la técnica de grupo focal, la cual es: "un espacio de opinión para captar el sentir, pensar y vivir de los individuos, provocando auto explicaciones" (Hamui-Sutton, 2012: 56) Pues son interpretaciones acerca de algún fenómeno social, como lo es la perspectiva de los actores sociales involucrados en el destino Ciudad Juárez y su influencia en las políticas públicas en turismo. Se convocó al gobierno (Gob), tercer sector (TS), empresa, instituciones de educación (IES) y profesionista en turismo (PT) en dos grupos focales diferidos en el periodo de febrero-junio de 2019.

Cada grupo focal es guiado por cinco preguntas: 1) ¿Las políticas podrían influir en la economía y cultura de Ciudad Juárez?; 2) ¿Cree usted que sería deseable pensar en políticas públicas para el desarrollo de Ciudad Juárez?; 3) ¿Cómo las políticas pueden beneficiar el turismo en Ciudad Juárez?; 4) ¿Qué políticas públicas conoce?; 5) ¿Cuáles podrían aplicarse al Turismo?

Los datos que emergen de los grupos focales, se trascriben de manera explícita y se procesan los testimonios con el software Atlas.Ti, el cual es un software que auxilia al análisis cualitativo de datos. Ya que permite la organización de datos, a través de la segmentación y codificación para la recuperación de significados. Se han identificado nodos con conexiones, asociaciones y articulaciones entre los participantes. Se ha agilizo mediante las frases clave y de esta forma generar conjeturas y supuestos de los fenómenos estudiado mediante el establecimiento de relaciones entre categorías (Muñoz-Justicia y SahagúnPadilla, 2017).

\section{RESULTADOS Y DISCUSIÓN}

La Tabla 1 muestra la participación de gobierno, tercer sector, empresa, instituciones de educación y profesionistas. Se aprecia desequilibrio en la contribución del ámbito gubernamental. Sin embargo, se logró la interacción una proporción más homogénea del tercer sector (TS), empresa, instituciones de educación (IES) y profesionista en turismo (PT) como actores interesados.

Tabla 1: Participación de actores e interesados

\begin{tabular}{|c|c|c|c|}
\hline No. & Participante & Cantidad & Porcentaje \\
\hline 1 & Gobierno & 1 & $6 \%$ \\
\hline 2 & Tercer Sector & 4 & $24 \%$ \\
\hline 3 & Empresa & 3 & $18 \%$ \\
\hline 4 & Instituciones de Educacion Superior & 5 & $29 \%$ \\
\hline 5 & Profesionista en Turismo & 4 & $24 \%$ \\
\hline & Total & 17 & $100 \%$ \\
\hline
\end{tabular}

Fuente: elaboración propia con base a trabajo de campo.

Como resultado de los grupos focales, se han obtenido puntos de vista, orientaciones y pautas de los actores participantes. Las cuales son parten de las preguntas guías, que han permitido configurar cuatro redes semánticas, con base a las expresiones vertidas y el análisis mediante el software Atlas. Ti. A partir de la primera pregunta ¿Las políticas podrían influir en la economía y cultura de Ciudad Juárez? Las políticas públicas son instrumentos que, a través de la coordinación de los 3 niveles de gobierno, buscan el beneficio social.

Realidad comentada por más de una vez por los participantes en los grupos focales, haciendo un especial hincapié en la continuidad, el seguimiento y la actualización que deben 


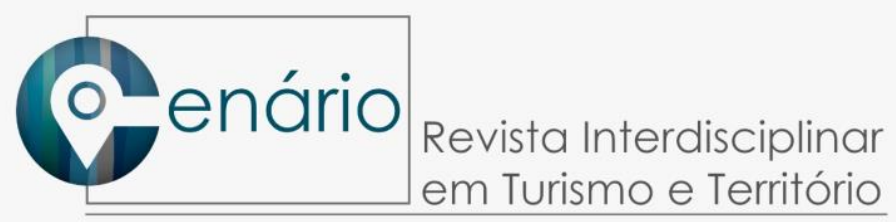

tener en busca de un desarrollo regional equilibrado que no solo vele por el aspecto económico, sino también cuide de lo social y ambiental. Pues el gobierno en lo general no ve el potencial del uso de las políticas. Pero mayormente por el gobierno municipal, pues en los diferentes periodos ha sido apático en su incorporación. Se consideran, que ideas del ámbito cultural y de turismo pierden su valor se, o se difuminan ante aspectos operativos (Castillo, Peñaloza y Tamayo, 2008). Pues se requiere inversión pública y privada: "La insuficiencia de fondos para satisfacer la demanda también puede reflejar una falta de priorización o enfoque estratégico para asegurar que el financiamiento se dirija a proyectos con el mayor potencial (OECD, 2017:27)".

La Figura 1, Influencia de las políticas públicas en la economía y cultura de Ciudad Juárez, muestra primordialmente los participantes de IES, Gob y TS. Los cuales coinciden en la importancia del correcto diseño y aplicación de políticas públicas. Por su influencia en la ciudad, pues al ser un destino internacional en un contexto binacional. La accesibilidad al destino es la situación en lo que se debe buscar una solución.

Figura 1. Influencia de las políticas públicas en la economía y cultura de Ciudad Juárez

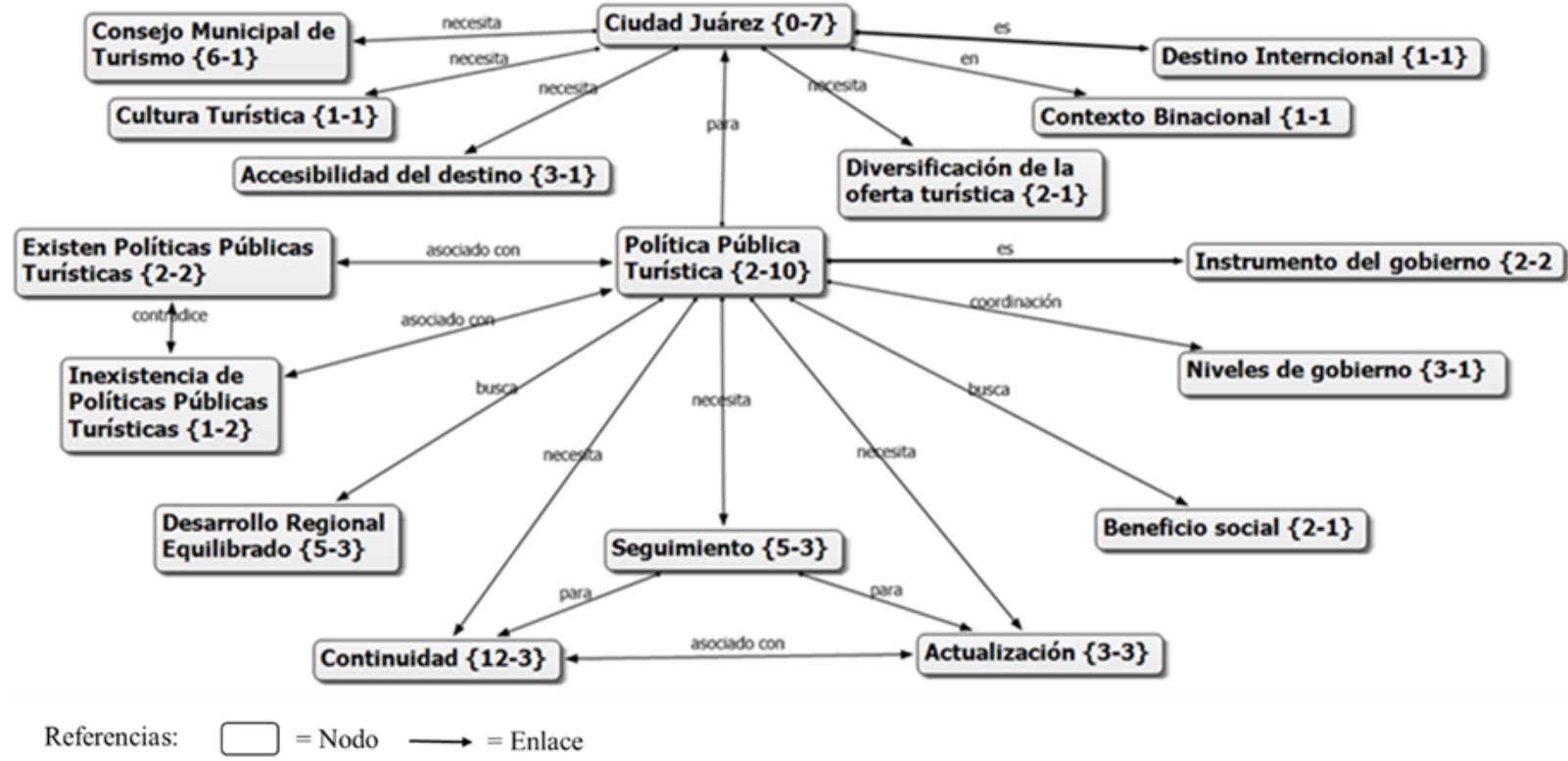

Fuente: Elaboración propia con base a trabajo de campo 2019, y Atlas.Ti.

La red semántica coincide con la postura de Monfort (2000). Cuando se refiere a las directrices y motivaciones existentes de quien depende la incorporación de la política pública. Ya que requiere, la gestión de estrategias, que permitan contar con planes, programas, así como esfuerzos legislativos, para el alcance de objetivos y metas previamente establecidos en un plan. Es así como, un criterio de la OECD (2017:29) se fundamenta en: Fortalecer el papel del Instituto Nacional del Emprendedor (INADEM) en la prestación de apoyos financieros y no financieros a las pequeñas y microempresas de turismo. Mejorar la capacidad financiera de las pequeñas y microempresas mediante la asistencia técnica y la capacitación.

Mediante la pregunta 2: ¿Cree usted que sería deseable pensar en políticas públicas para el desarrollo de Ciudad Juárez? Se genera la Figura 2 sobre las PPT para el desarrollo de Ciudad Juárez, el diagrama representa la coincidencia de los participantes sobre la relevancia de las políticas públicas para el desarrollo de la ciudad. La cual radica en que integra al gobierno, a la sociedad, a las asociaciones civiles, la academia y por supuesto a la 


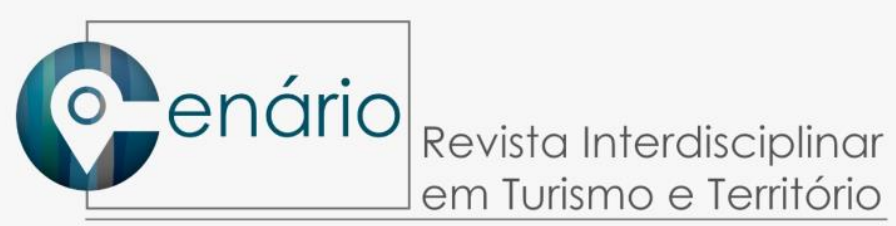

iniciativa privada. En este sentido la finalidad se centra en un desarrollo local-regional equilibrado. Entre los hallazgos, la importancia que tiene el gobierno para la difusión de las PP, y la necesidad de su aplicación para enfrentar problemáticas. Ejemplo de lo anterior: la rotación del personal y la capacitación. Esta última destaca, pues se enfatizó la crítica al entrenamiento a las que no se les da el seguimiento adecuado por parte del gobierno como del sector privado.

En la red (Figura 2) se observa las prioridades de la PP en el turismo según los actores sociales partícipes. Indican por una parte la necesidad de crear apego a la ciudad en la población, y con ello generar el compromiso con su sociedad y además de integrar la Triple Hélice (Gobierno, Academia e Iniciativa Privada). A más del énfasis en la participación integrada para la creación, promoción y continuidad del apego. Pues, este escenario garantizaría su continuidad pese a los cambios del gobierno y la migración al destino.

Figura 2. Políticas Públicas para el desarrollo de Ciudad Juárez.

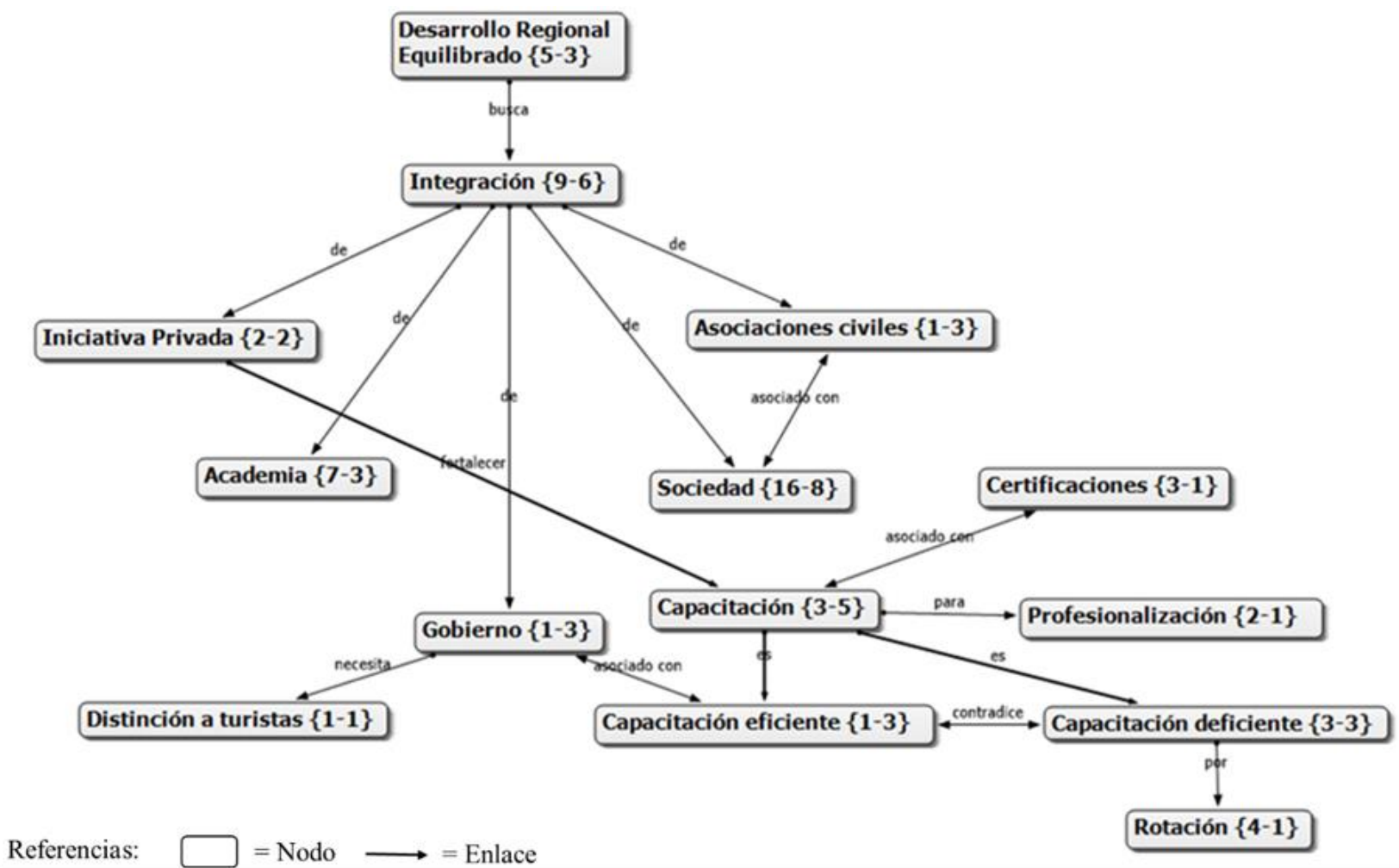

Fuente: Elaboración propia con base a trabajo de campo 2019, y Atlas.Ti.

También, en los grupos focales fue evidente la complejidad del turismo como actividad. Fue posible identificar también: [...] nuevas interpretaciones sobre las relaciones culturales en zonas y actores fronterizos [...], en el que se acentúan los rasgos conectivos y los puentes culturales, enfatizando los elementos comunes más que las diferencias (Valenzuela, 2019:53). Por tanto, al involucrar diferentes actores e interesados, han aportado multidisciplinarmente.

Creo que se han integrado muy activamente los elementos de comercio del segmento hotelero, del segmento restaurantero inclusive a nivel municipal se hizo un programa para profesionalizara los cuerpos policiacos y de vialidad para que tuvieran una 


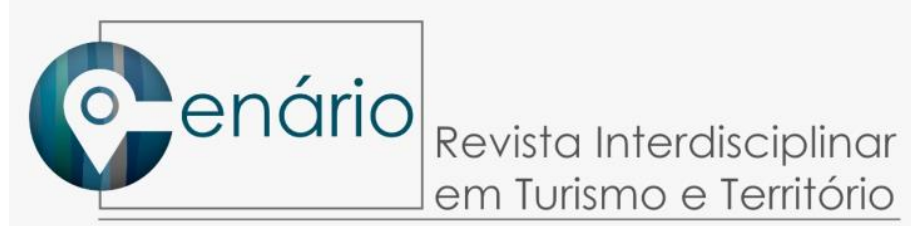

empatía [...], creo que por ese sentido vamos dictaminando bien porque es la política. Siller, 2019, Gob.

La pregunta 3. ¿Cómo las políticas pueden beneficiar el turismo en Ciudad Juárez? Provoca la reflexión sobre el destino de Ciudad Juárez. Al ser como un destino con imagen deteriorada. Esta circunstancia condiciona su desarrollo como actividad turística. En este sentido se requiere el fomentar la cultura y el reconocer el patrimonio local en la comunidad. Estas acciones auxiliarán al fortalecimiento del sentido de pertenencia. Pues llegan al destino constantemente oleadas de migrantes en forma constante. Esta situación daña el sentido de pertenencia y por lo tanto el cuidado del mismo. Las PPT son una oportunidad ya que pueden generar programas que den originen a una educación integral del aprecio del patrimonio cultural local. Pues, mediante la enseñanza se infunde la riqueza cultural e histórica con la que cuenta la comunidad.

En la Figura 3, pauta la actividad de los actores e interesados a favor de la configuración de Consejo Municipal de Turismo. Los participantes indican la oportunidad de trabajo consultivo. Escenario propicio para recibir propuestas de académicos, sector privado o la sociedad en general. Además, señalan la ocasión para la diversificación del destino a través de tendencias como el Turismo Médico y la necesidad del mercado de atracción de visitantes nacionales, pero también de ampliar las estrategias para la atracción internacional. Aunque se requiere identificar las ya existentes. El correcto uso de estratagemas públicas, potencializa cambios significativos al destino y de beneficio.

Figura 3. Políticas Públicas para el beneficio al turismo en Ciudad Juárez.

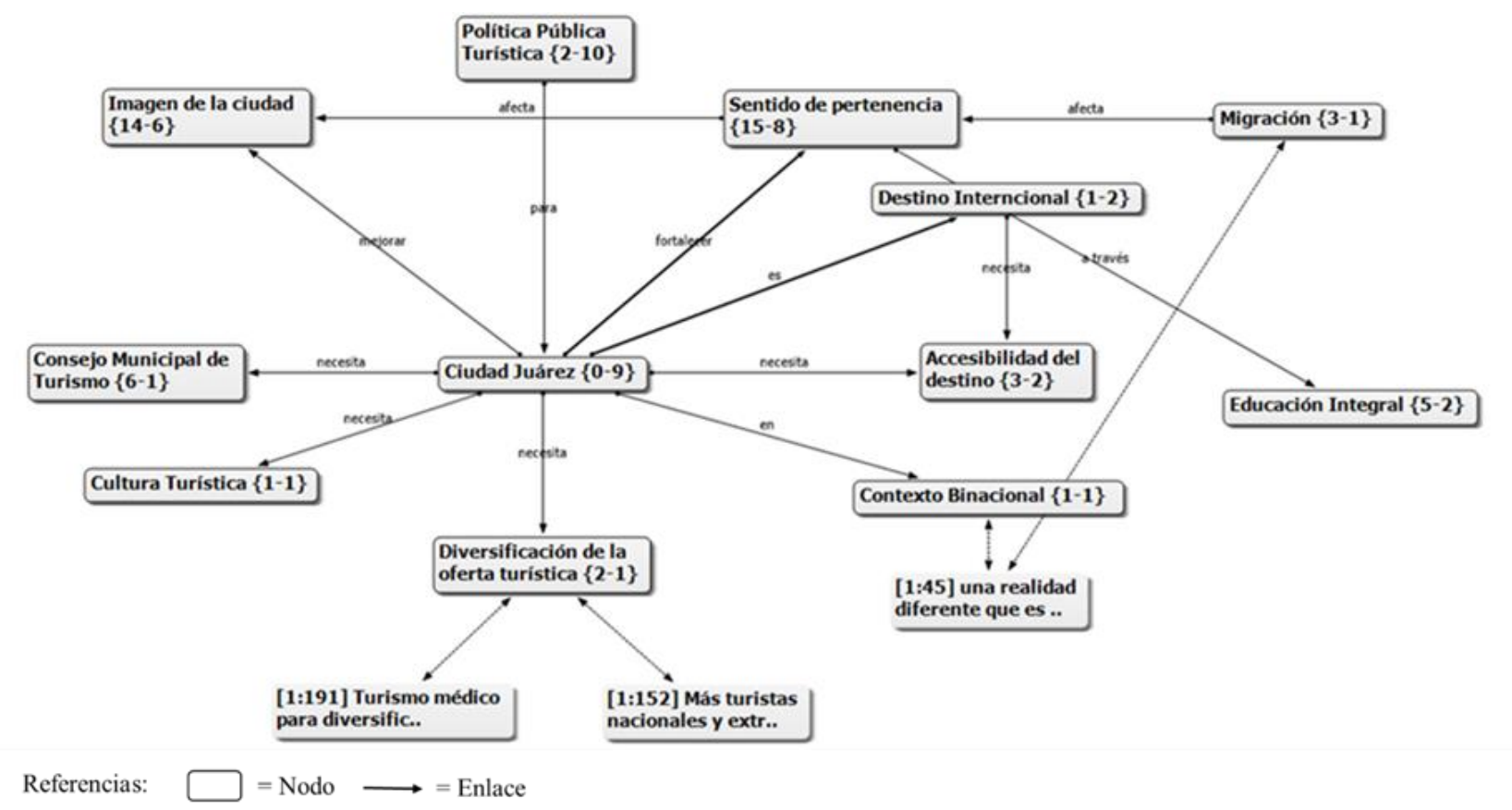

Fuente: Elaboración propia con base a trabajo de campo 2019, y Atlas/ti.

En la red los participantes conciben la importancia de fomentar el cuidado y la historia de la ciudad desde primaria hasta universidad, con la finalidad de lograr el apego a la ciudad y a su patrimonio cultural. Además, se reconoce el trabajo de las distintas asociaciones que fomenta a través de publicidad positiva la inclinación por la ciudad. 


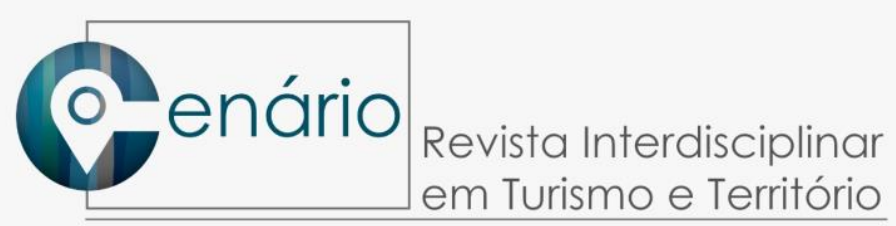

[Comente con] las regidoras del municipio que debíamos establecer una placa o alguna identificación que se le pueda dar al turista para que en un momento determinado no sea interpelado tanto por policías como por transito que en cuanto le ven placas extranjeras rápidamente los paran y les inventan unas cosas. Siller, 2019, Gob.

El cuestionamiento 4, pregunta ¿Qué políticas públicas conoce? Los participantes mencionan en repetidas ocasiones, las certificaciones y programas del gobierno municipal y estatal ha promovido, como lo es el Taxi Amigo (Figura 4), el programa certifica a los taxistas, en su labor por los conocimientos sobre la información turística y su intención es mejorar el servicio tanto a locales como a los visitantes.

Figura 4. Conocimiento de políticas públicas.

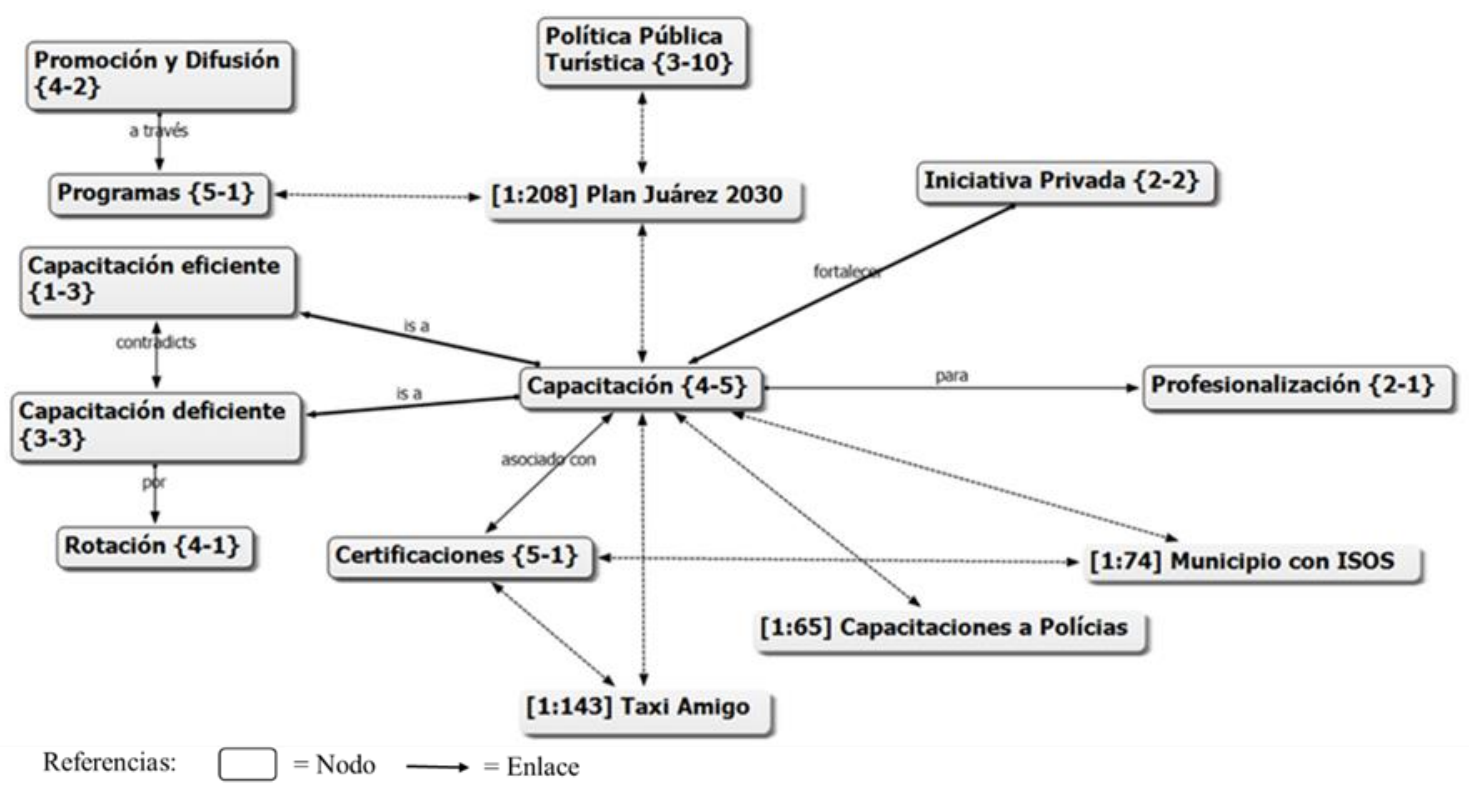

Fuente: Elaboración propia con base a trabajo de campo 2019 y Atlas/ti.

Por otra parte, la capacitación a la policía municipal también marca un referente en los datos cualitativos. El municipio mediante este esquema está comprometido en cumplir los parámetros y metas establecidas en el Plan Juárez 2030; aunque la mayoría de los partícipes se mostraron escépticos. Sin embargo, a los participantes no les fue posible identificar políticas públicas que se encuentren relacionadas con el apoyo al patrimonio cultural. Situación preocupante en una comunidad que su pasado se encuentra ligada a la historia y la actividad turística. Ante la falta de aportes en la pregunta 4, hace suponer el desconocimiento de la existencia de PPT o su inexistencia, lo que presupone por qué no se están considerando o en su caso aplicando correctamente.

[...] yo considero que también la sociedad influye mucho en que no se cumplan esas políticas. Medina, 2019, PT

Es un escenario en el que no se puede exigir al gobierno de un seguimiento adecuado a una situación que se desconoce.

Creo yo que no pudiera llamarse fracaso, pero la falta de alcance y de penetración de las políticas públicas en turismo y en muchas otras áreas como [...] se debe a que no se ha implementado un modelo de gobernanza en turismo en donde se incluyan los 4 actores (la sociedad civil, las universidades, el gobierno y la iniciativa privada) González, 2019, IES.

En la Figura 5 Sentido de pertenencia y su importancia para las políticas públicas nace de la pregunta 5. ¿Cuáles podrían aplicarse al Turismo? Las respuestas esbozan la postura sobre las políticas públicas en Ciudad Juárez. Es decir, se requiere un enfoque en 


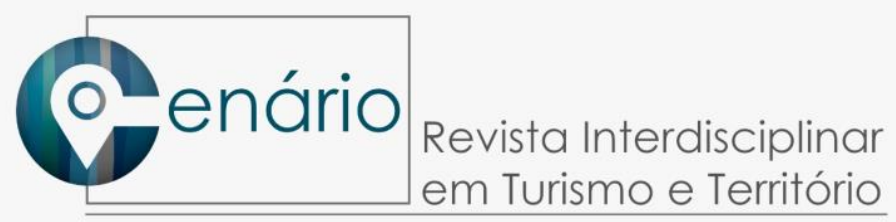

programas que incentiven la pertenencia, como la identidad en los habitantes de la ciudad. Al fortalecimiento del vínculo entre la cultura y ciudad, pues se espera un reflejo positivo en la sociedad. Sin embargo, los esfuerzos han sido tenues y aislados en los programas asociados como: "Amor por Juárez", "I love Juárez" y otras campañas como "siéntete orgulloso" y "si vives en Juárez, eres de Juárez". Las aportaciones puntualizan el valor que tiene el papel de la sociedad en el despliegue de políticas públicas y cómo estás, es la sociedad que debe asumir su responsabilidad sobre sus acciones en la mejora por la imagen del destino. Pues: "Está claro que las acciones no son lineales, ya que la gestión pública sigue siendo difusa (Gayer, 2017: 22)".

Figura 5. Sentido de pertenencia y su importancia para las políticas públicas

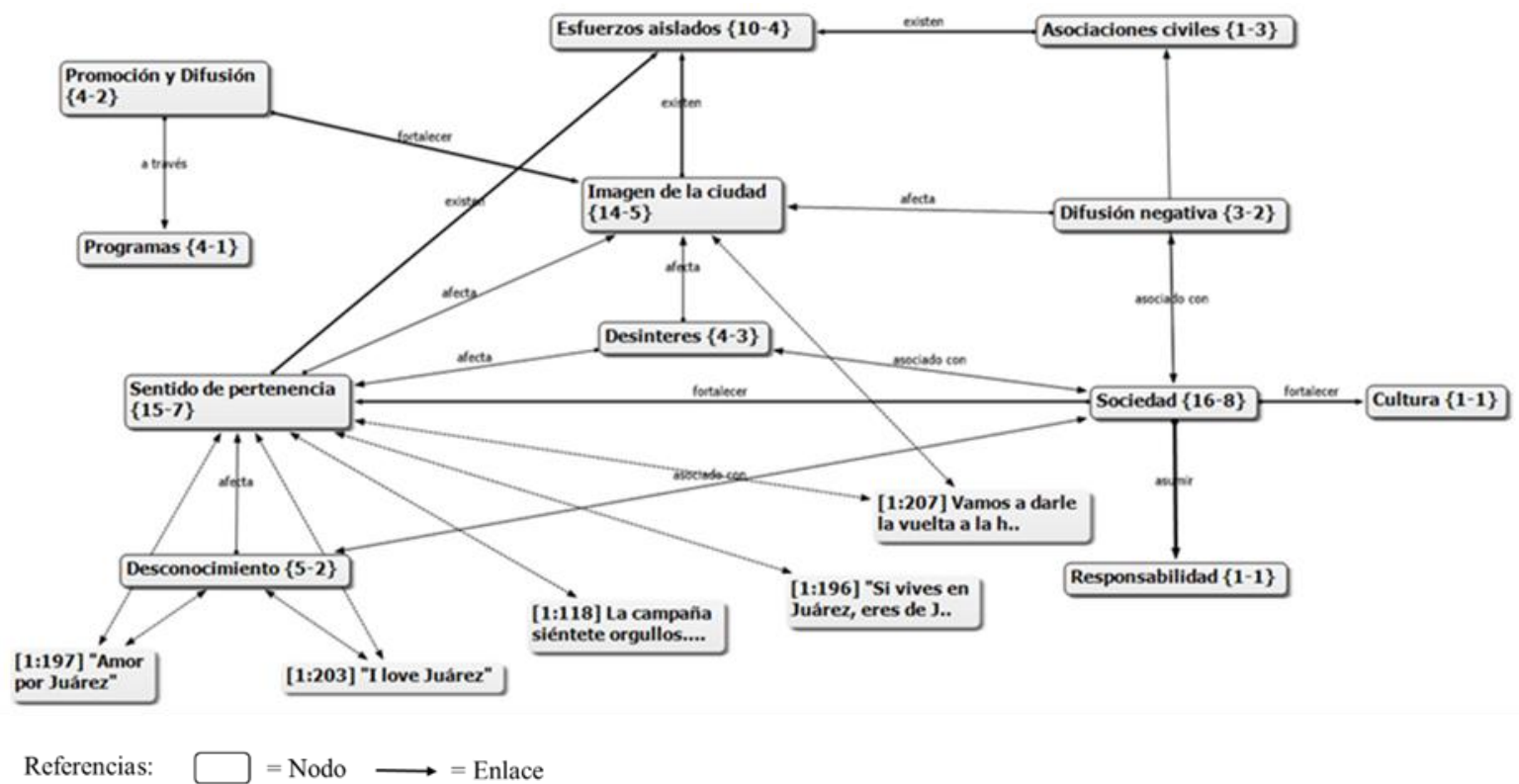

Fuente: Elaboración propia con base a trabajo de campo 2019, y apoyo de Atlas/ti.

Como eje central en esta red semántica, se observa que la sociedad, debe involucrarse en la creación, promoción y seguimiento de la política pública a través del desarrollo de una cultura turística. Razón de peso la relación binacional y su contigüidad cultural, en cuanto que: "Las relaciones fronterizas y transfronterizas son polisignificantes, ya que articulan procesos complejos y contradictorios, [en] las áreas fronterizas que son espacios geográficos y culturales conformados entre dos o más naciones (borders) (Valenzuela, 2014:18)". Dicho de paso, que revitalice el interés y el compromiso para un mayor alcance de estas. Es decir, la red muestra como la sociedad debe ser incluida, con la finalidad de que las acciones cumplan su propósito. Ya que su intención es el establecer PPT que promuevan el desarrollo del destino, al fomentar la educación, la historia de CJS, y trabajar en la preservación del patrimonio cultural creando conciencia y compromiso. Así como el cuidado que se debe tener hacia la misma.

[...] conocer nuestra historia, crear talleres o hacer algo un poco más informal pero informativo, invitar a la comunidad a las sociedades en general, niños, adultos, hacer sesiones de películas o fotografía donde les den un tour y les den a conocer lo que es la historia de la ciudad para primero conocer y luego protegerlo. Villa, 2019, PT.

[...] cuanto estaba el Presidente municipal Galindo que decía "Si vives en Juárez, eres de Juárez" y esa campaña se promovía 24/7 para que la gente tuviera un sentido de identidad, pero eso lo tienen que tomar directamente el gobierno, tiene que conducir esta campaña. Álvarez, 2019, IES.

CENÁRIO|ISSN 2318-8561|Brasília|V.8, n. 14|130 - 143|Jun. 2020|

DOI: $10.26512 /$ revistacenario.v8i14.31174 


\section{CONCLUSIONES}

México en su postura de política pública turística ha mostrado congruencia en sus planes y programas. Algunos autores las consideran bien articuladas, pues tienden a la estimulación de inversión, como al crecimiento. Este crecimiento se visualiza más el contexto de un desarrollo equilibrado y sostenible. Ejemplos de lo anterior se encuentran primordialmente en los destinos de playa, como Ixtapa Zihuatanejo, Los Cabos o Cancún. Más la existencia del Programa de Pueblos Mágicos.

En el caso de CJS la situación se torna en postergación, es decir, ya sea por la falta de voluntad política, de información o conocimiento de la actividad. Queda evidente la ausencia de las políticas públicas en turismo. Y resulta inquietante el escenario para la comunidad, en comparación con los entornos planificados. Ante esta insuficiencia de políticas la aproximación al fenómeno, la teoría del actor-red ha permitido una intervención al fenómeno no solo al objeto como el qué, adicionando la desvinculación del ámbito antropocéntrico que propone la sociología. La interacción siguiendo la teoría de ANT cedió a los grupos focales, un contraste ante la necesidad de políticas públicas específicas sobre patrimonio cultural y turismo para Ciudad Juárez. Pues limita el desarrollo socioeconómico y en términos de presenvación y conservación. Como es la postura de la teoría, el acercamiento a los actores, permitió la identificación de descripciones de los escenarios a través de las preguntas guías.

Sin embargo, el aliciente ante la situación son los hallazgos que muestran ideas para promover el patrimonio cultural en CJS. El interés que se reflejan en las aportaciones no solo busca enaltecerlo, pero igual resguardar e incluso promoverlo. Por otra parte, es evidente el desconocimiento de la existencia de PPT por los participantes, tanto de gobierno, instituciones de educación superior, iniciativa privada y tercer sector. Un hallazgo en la red semántica es la mención Consejo Municipal de Turismo, lo que permitirá la contribución de actores e interesados. Por tanto, situación se presentarán oportunidades por la interrelación del turismo con otras áreas económicas y políticas. Mediante los representantes del gobierno municipal, empresarios y académicos, que conformaran un grupo multidisciplinario, responsables en la toma de decisiones y acciones consensuadas en pro de las PPT.

Finalmente, los beneficios de las políticas públicas en turismo serán evidentes para el caso de CJS en la medida de ser parte del destino y los actores e interesados comprendan lo trascendente del binomio patrimonio cultural y su relación inexorable con el turismo.

\section{REFERENCIAS}

Arcoraci, E. (2009). Economía y políticas turísticas. unidad VIII: Política Turística Recuperado el 5 septiembre de 2019, en http://www.utntyh.com/wpcontent/uploads/2009/11/UNIDAD-8-.POLITICA-TURISTICA.pdf.

Arnaiz Burne, S. M. y Dachary A. C. (2009). Geopolítica, recursos naturales y turismo. Una historia del Caribe mexicano. Universidad de Guadalajara. Puerto Vallarta, Jalisco, México.

Bezerra Cavalcante, R, da Silva Esteves, C. J., Calisto de Assis Pires, M., Dias Vasconcelos, D., de Melo Freitas, M., 6 Sávio de Macedo, A. (2017). The actor-network 


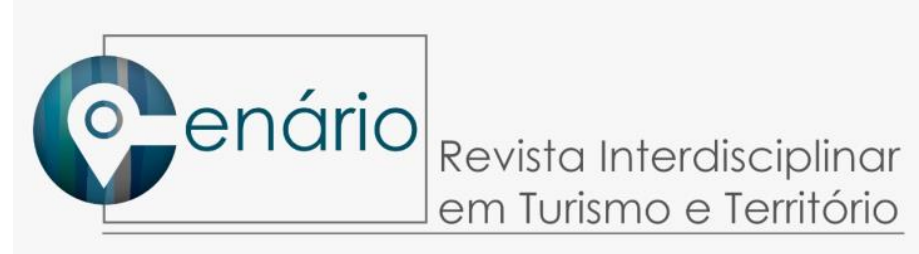

theory as a theoretical methodological framework in health and nursing research. Texto Contexto Enferm, 26(4): e0910017.

Castillo Nechar, M., Peñaloza Suarez, L. y Tamayo Salcedo, A.L. (2008). Las Políticas Turísticas Culturales en el Estado de México. Gestión Turística, núm. 9, pp. 87-106.

Castillo, M., Peñaloza Suarez, L. y Tamayo Salcedo, A.L. (2008). Las Políticas Turísticas Culturales en el Estado de México. Gestión Turística, (9), 87-106.

Edgell, David L. and Jwanson, Jason R. (2013). Tourism Policy and Planning. Routledge, USA and Canadá, Elsevier.

Enríquez, M., Osorio, M., Castillo, M. y Arellano, A. (2012). Hacia una caracterización de la Política Turística. Pasos. Revista de Turismo y Patrimonio, 10 (3), 417-428.

Gallegos, O. y López A. (2004). Turismo y estructura territorial en Ciudad Juárez, México. Investigaciones Geográficas, (53), 141-162.

Gayer, P. (2017). Políticas públicas em turismo de eventos: instrumentos normativos de apoio ao desenvolvimento do setor. CENÁRIO, Brasília, V.5, n.9, pp.12-22.

González Herrera, M. (2012). Cultura Turística de la Población Local Anfitriona del Turismo. España: Editorial Academia Española.

López Gómez, D. \& Tirado, F. (2012). Teoría del Actor-Red: Un pragmatismo contemporáneo. En Teoría del actor-red: Más allá de los estudios de ciencia y tecnología (pp. 1-16). Barcelona: Ed. Amentia.

Madagán Díaz, M. y Rivas García, J. I. (2012). Estructura, Economía y Política Turística. Septem Ediciones. España.

Madrid, F. (2015). La sostenibilidad en la política turística mexicana. Pasos. Revista de Turismo y Patrimonio Cultural, 13(6), 1301-1313.

Magaña-Carrillo, I. (2009). La política turística en México desde el modelo de calidad total: un reto de competitividad. Economía, Sociedad y Territorio, volumen IX, núm. 30, pp., 515544.

Meyer Montenegro, L. and Bulgacov, S. (Jan/Mar. 2014). Reflections on Actor-Network Theory. BAR, Rio de Janeiro, v. 11, n. 1, art. 6, pp. 107-124, www.anpad.org.br/bar.

Molina, R., Ochoa M. y Leco, C. (2014). Políticas públicas y factores que determinan la competitividad turística de Morelia, México y de Alcalá de Henares, España. Revista CIMEXUS, IX (2), 49-68.

Monfort, V. (2000). La política turística: una aproximación. Cuadernos de Turismo, 6 (2), pp. 7-27.

Montañés Duato P. (2012). Inteligencia Política; El Poder Creador en las Organizaciones. Madrid, España: Prentice Hall. 


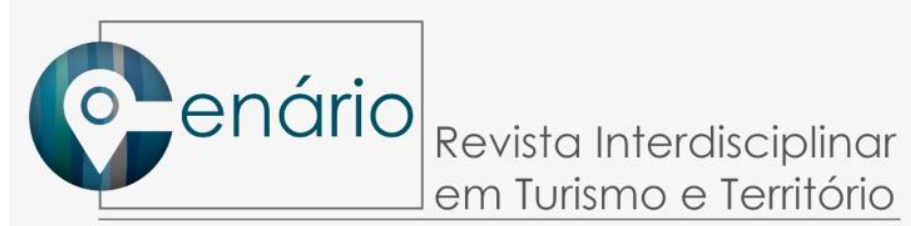

Morales, G. (2015). Teoría del Actor-Red y turismo. Dos casos de estudio en Perú y Argentina. 1er. Congreso Latinoamericano de Teoría Social en Buenos Aires.

OECD (2017). Estudio de la Política Turística de México. Resumen Ejecutivo, Evaluación y Recomendaciones. México: Secretaría de Turismo de México. Recuperado en https://www1.oecd.org/industry/tourism

PND 2013-2018 (2013). Programa Nacional de Infraestructura 2014-2018. Recuperado de http://www.dof.gob.mx/nota_detalle_popup.php?codigo=5342547

Rodríguez, M. y Zamora, E. (2009). La colaboración entre los actores turísticos en ciudades patrimoniales. Reflexiones para el análisis del desarrollo turístico, Pasos. Revista de Turismo y Patrimonio Cultural, 7(2), 219-238.

Ruiz Chávez O. (2008) Turismo: Factor de Desarrollo y Competitividad en México. Centro de Estudios Sociales y de Opinión Publica, (46), 1-33.

Staudt, K. y Vera, B. (2006). Mujeres, políticas públicas y política: los caminos globales de Ciudad Juárez, Chihuahua-EI Paso, Texas. Revista Región y Sociedad. XVIII (37), 127-172.

Urrutia, J. y Cuevas, T. (2016). Redes empresariales en el sector turismo y servicios para la mejora de competitividad en Ciudad Juárez, Chihuahua, México: Caso parque central Hermanos Escobar y Pymes Aldeñas. Cuaderno de Turismo, (37), 421-436.

Valenzuela, J. (2014). Transfronteras y límites liminales. En Valenzuela, J. (Ed.), Transfronteras. Fronteras del mundo y procesos culturales (pp.17-44). Tijuana, México: El Colegio de la Frontera Norte, A.C.

Valenzuela, J. (2019). Transfronteras: la condición fronteriza y los estudios de frontera. En Giménez, G. y Gutiérrez, N. (Ed.), Las culturas hoy (pp.51-71). Ciudad de México, México: Universidad Nacional Autónoma de México.

Velasco, M. (2011). La Política Turística. Una arena de acción autónoma, Cuaderno de Turismo, (27), 953-969.

Velázquez García, M. A. (2013). La formulación de las políticas públicas de turismo en México. El caso del programa federal "Pueblos Mágicos" 2001-2012 Diálogos Latinoamericanos, núm. 21, diciembre, 2013, pp. 89-110.

Villar, A. (2009). Políticas públicas en Turismo. En Noemí Wallingre y Alejandro Villar (Compiladores) Desarrollo y gestión de destinos turísticos. Políticas y estrategias. Argentina: Universidad Nacional de Quilmes Editorial.

\section{Fuentes electrónicas}

https://indexjuarez.com/wp-content/uploads/2018/04/Marzo-2018-3-2.pdf

https://problemasdelafronteranorte113953.wordpress.com/2015/11/02/programa-nacionalfronterizo-pronaf/ Consulta: 5 septiembre de 2019. 
